\title{
Sciendo
}

DOI: 10.1515/aon-2019-0004

\section{DYNAMIC PROBABILITY FIELDS FOR RISK ASSESSMENT AND GUIDANCE SOLUTIONS}

\author{
Edwin Williams \\ The University of Southern California \\ 920 Downey Way, BHE B13, Los Angeles, CA 90089, USA \\ email: edwinwil@usc.edu \\ Yan Jin \\ The University of Southern California \\ 3650 McClintock Ave, OHE 400D, Los Angeles, CA 90089, USA \\ email: yjin@usc.edu
}

\begin{abstract}
Standard Guidance, Navigation, and Control (GN\&C) systems take state data from a navigation system and create a trajectory that minimizes some a-priori determined cost function. These cost functions are typically time, money, weight, or any general physically realizable quantity. Previous work has been done to show the effectiveness of using risk as the sole objective function. However, this previous work used Poisson distributions and historical estimates to achieve this goal. In this paper we present the situation-risk assessment (SRA) method contained within the intelligent situation assessment and collision avoidance (iSC) platform. The SRA method uses data clustering, and pattern recognition to create a historically based estimate of guidance probabilities. These are then used in data driven, dynamic models to create the future probability fields of the situation. This probability, along with the other agent's goals and objectives, are then used to create a minimum risk guidance solution in the nautical environment.
\end{abstract}

Keywords - GNSS, Galileo, EDAS, tracking, firefighting, multisensor, fusion, communication optimization

\section{INDRUCTION}

Guidance, Navigation, and Control (GN\&C) systems have had the same general structure since they were first developed by Goddard. This GN\&C loop is shown in Figure 1, below. In this system sensor errors are filtered through a stochastic navigation algorithm to develop the best system state estimate. A plan that was developed by teams of engineers is then calculated through the guidance algorithm. Finally this plan is executed through the system controller.

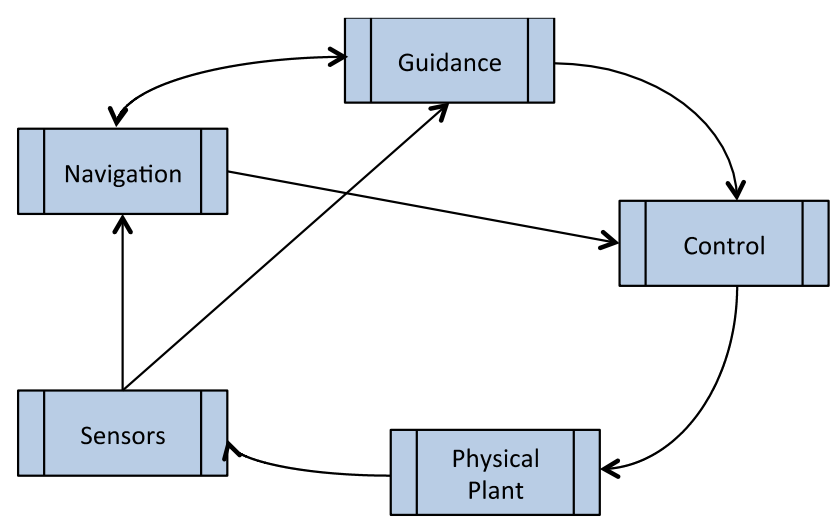


Figure 1. Standard guidance, navigation, and control system. Source: authors.

Throughout this paradigm the designer imposes some a-priori situation analysis [Ullman, 2010]. Teams of engineers develop cases and clauses that are triggered in the guidance algorithm as certain navigational estimates are developed. The robustness of a guidance solution is based on how many situation types a system will be able to account for. It must be noted that this is robustness not in the control or navigation space, which has a fully developed theory [Lavertsky and Wise, 2013], but in the guidance solution itself.

Previous work [Williams and Jin, 2018] in this area has proposed a paradigm in which the uncertainty of the entire data becomes intrinsic to the guidance solution developed. This brings a multitude of work [McHenry et al, 1979], [Fossen, 2002], [Steinfeldt et al, 2010], [Kaiser et al, 2010], etc on navigation uncertainty to the realm of guidance solutions. Specifically setting up a methodology to answer the question of how to handle a situation or dataset that does not meet any of the a-priori assumptions of the system engineers.

This paper expands upon the previous work of risk determination from an arrival model, and instead utilize the direct probabilities and statistics of the situation. The specific problem addressed in this is the nautical problem of ship collision avoidance. Standard collision scenarios used to train ship captains will be used to assess the efficacy of the proposed methods. It needs to be noted that the methods to arrive at the statistical probabilities are not discussed in this paper [Williams and Jin, 2019], but instead what the effects of this change are. Also used in the development of these results are a formal optimal guidance solution.

\section{BACKGROUND}

\section{A. Situation Awareness}

From an engineering standpoint there are two (related) schools of thought on situation, situation awareness (SAW), and situation assessment (SA). The first of these views is the method proposed by Endsley [Endsley, 1995] on situation awareness. Endsley proposed that SAW is developed in through three levels. The first is determining the entities and states in the environment. The second level is understanding the relationships between those entities. Finally the third is the ability of an agent to project that situation into the future. This model is shown graphically Figure 2, below.

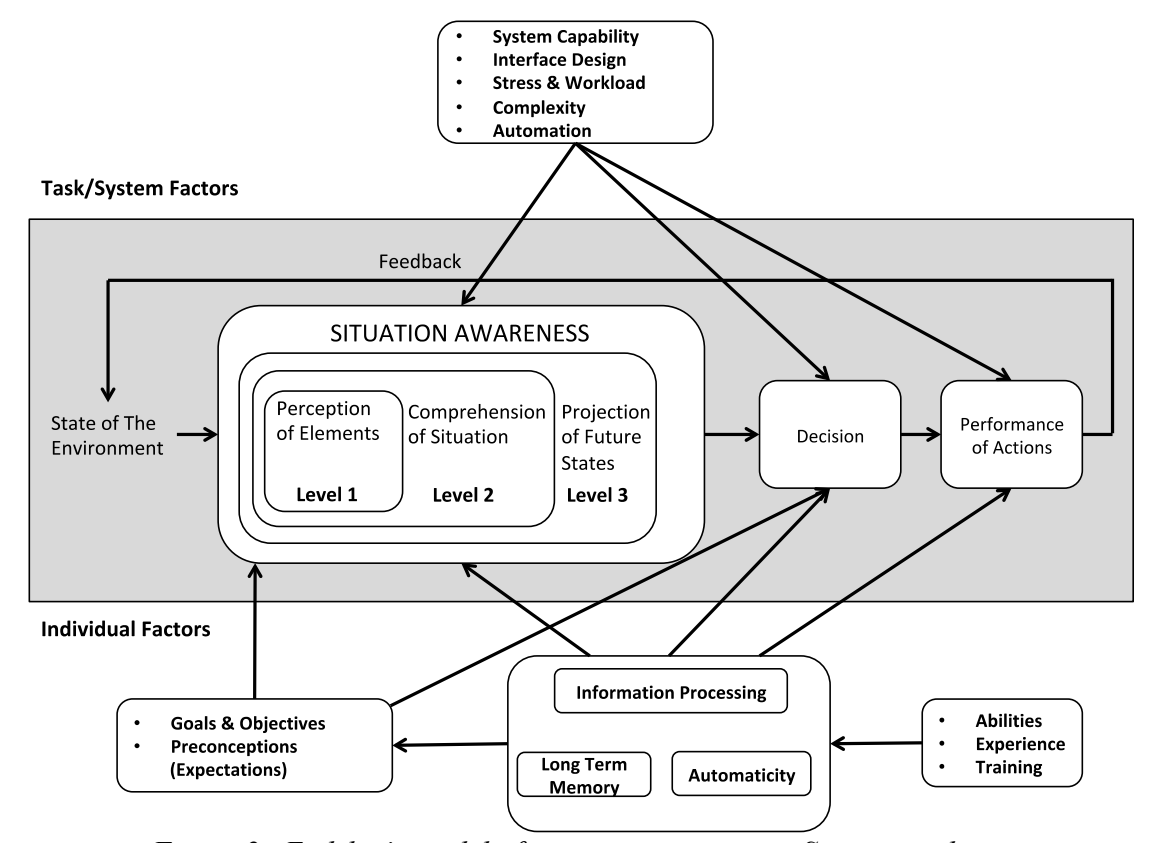

Figure 2. Endsley's model of situation awareness. Source: authors.

Endsley's model was initially developed for human cognition, and not necessarily for autonomous systems. However, Endsley and others have updated this cognition method to include multiple agents and system 
situational awareness [Endsley, 1998], [Endsley, 2017]. It should be noted that Endsley's model resembles the standard guidance, navigation, and control loop. The main difference is that SA views allow for a broader scope of data, beyond standard navigation state and sensor data.

The second direction comes from the ontological build up of SAW and SA through the sensor fusion community. This begins with the work of Barwise [Barwise, 2006] and later Devlin [Devlin, 2006] on the philosophical basis of situation and situation awareness. The ontology based SAW [Kokar et al, 2009] was developed as an object oriented data model completed with a UML based diagram. The use of predicate calculus and infons through the use of object oriented data development allows for the development of autonomous SA.

The situation assessment model and a traditional GN\&C system can be combined to have the flow of information be that shown in Figure 3, below.

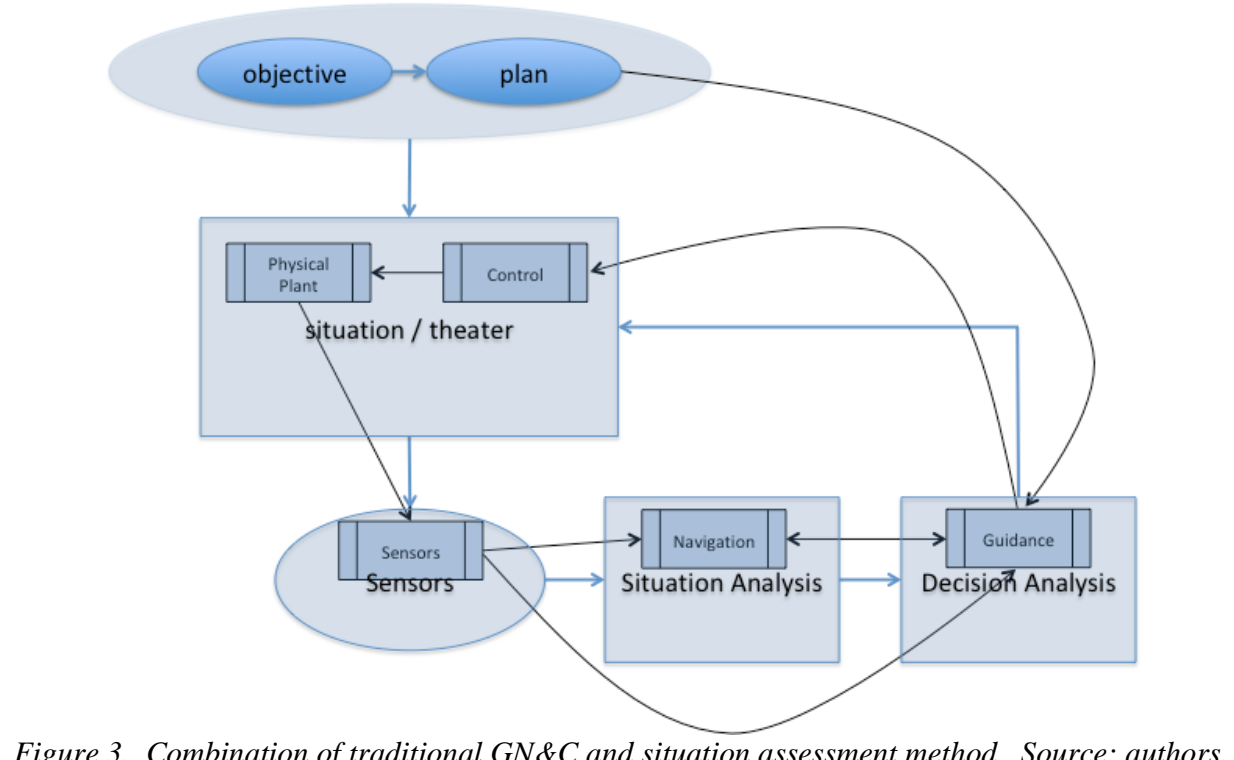

Previous work in this area [Williams and Jin, 2018] stressed the need to correctly identify our agents' goals. This will allow the system to classify the information into a correct situational assessment. For the nautical collision avoidance scenarios the following goals/objectives will be used:

1. Do not collide with anything

2. Get to the waypoint

3. Avoid changes to the optimal power

4. Do not roll the ship

\section{B. Trajectory Optimization}

The work that has been done on risk minimization in the past has used a greedy approach. Since it was a state arrival based model of risk, the arrival in the future was pre-built into the analysis of the system risk. This allowed for a simple greedy algorithm to determine the current guidance decision [Kutsuna et al, 2018]. However, since the current collision risk model is based upon the dynamics of the system and the decision making statistics a full trajectory analysis must be used.

This is accomplished by turning the trajectory optimization problem into a multivariable design optimization problem. Current systems use a pseudospectral approach [Ross and Karpenko, 2012] to solve this. However, it has been observed in tests that for the nautical environment a single shooting method is sufficient. 
The simple single shooting method works for the risk minimization problem since all of the constraints are held within the objectives and are part of the risk minimization itself. So the traditional optimization problem shown in equation (1) has less external constraints and is thus easier to converge.

$$
\begin{gathered}
\min f(\vec{x}, \vec{u}) \\
\text { s.t. } \frac{d \vec{x}}{d t}=g(\vec{x}, \vec{u}),|\vec{u}| \leq \vec{c}, \vec{x}(\tau) \neq \vec{\Xi}(\tau)
\end{gathered}
$$

The reformulated optimization problem (equation (2)) has fewer external constraints. So standard design optimization methods allow for rapid convergence [Vanderplaats, 1984].

$$
\begin{aligned}
& \min R(\vec{u}) \\
& \text { s.t. }|\vec{u}| \leq \vec{c}
\end{aligned}
$$

\section{RISK MODELING}

As was stated in Section 2-A, an agent's objectives are what define that agent's risk. As such there are four different possible failure points for the nautical situation. The agent needs to go to the waypoint without colliding with anything, using too little or too much power, and also avoid rolling the vessel.

Each component of the total instantaneous risk is itself a percent chance of failure for that goal/objective. In the non-dynamic risk equations ((3), (5), (6)) there are scaled parameters. These parameters are determined through expert analysis or least squares fitting. Specifically the iSC system accesses data within a database and determines these coefficients from observed events in situation dependent categories. A discussion of this is provided in another work [Williams and Jin, 2019].

A diagram of the iSC system can be seen in Figure 4. This paper focuses on the Situation Assessment and Operation Planning portion of this system. However, much of the work to accomplish this path planning is needed to be done before hand. The statistical analysis and coefficient determination are done in the Data Collection and Knowledge Management portions of the system.

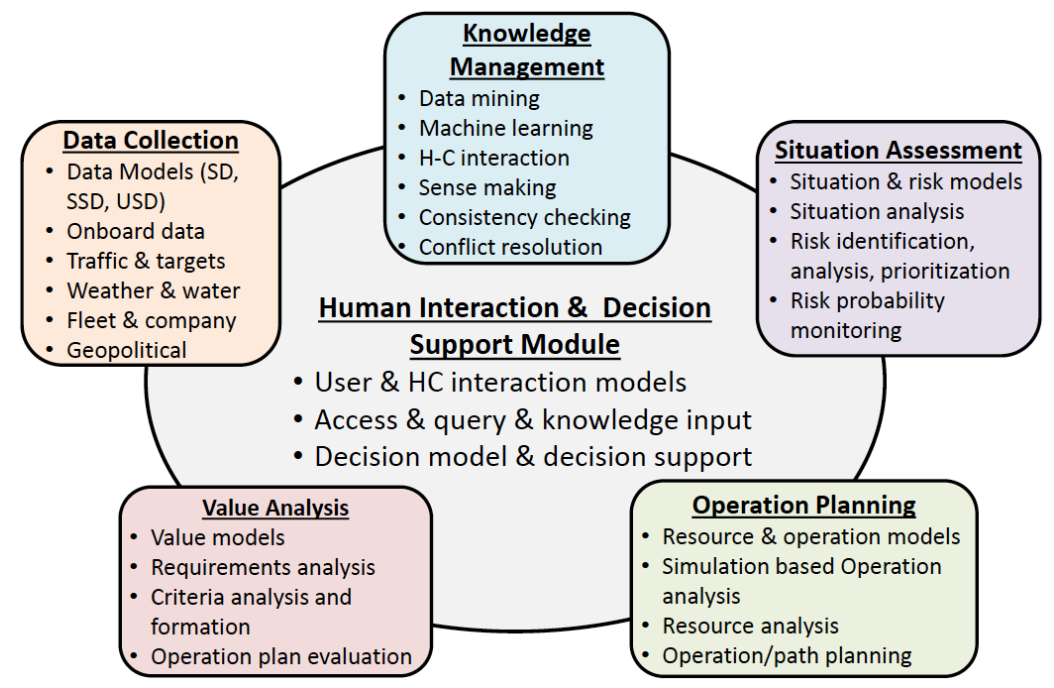

Figure 4. iSC system architecture. Source: authors.

For the purposes of this paper the system flow is shown in Figure 5. When the system has identified the objects in the environment the Knowledge Management module is queried to retrieve the best estimate of decision probabilities and dynamic response values. These are then used to propagate the probabilities of the system state forward in time (for purposes of this paper this is 10 minutes in the future). The optimal trajectory 
is then determined that minimizes the total risk. After a settling time (for this paper that is 30 seconds of simulation time) the system is re-evaluated and a new optimized trajectory is developed based upon the latest system state.

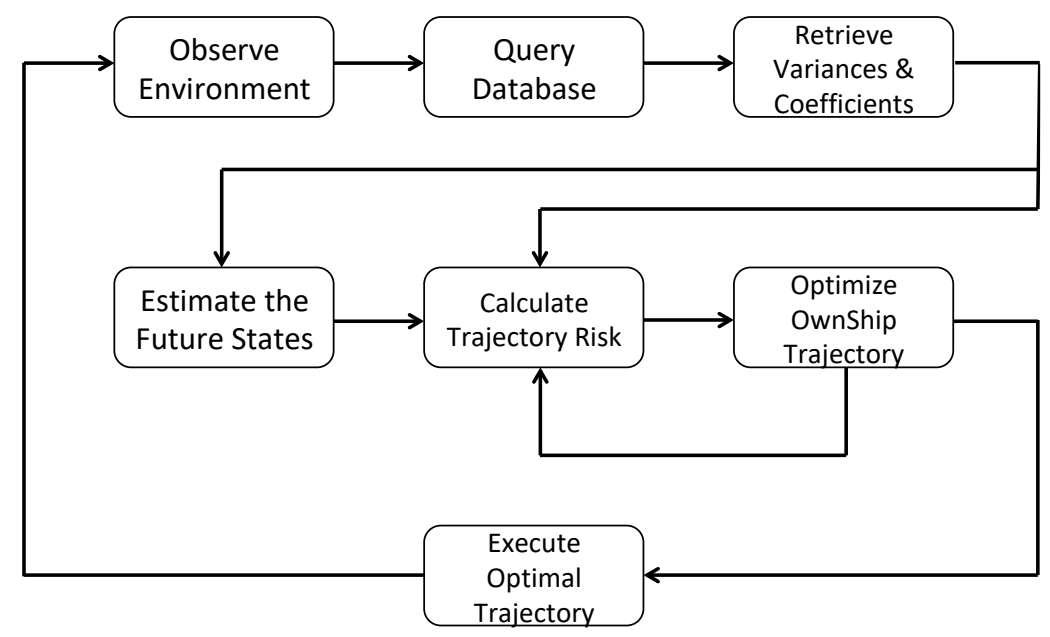

Figure 5. Data flow for the SA and OP portion of the iSC. Source: authors.

\section{A. Waypoint Risk}

If there were no other goals, the best solution would simply point OwnShip's velocity vector in the direction of the way point and go as fast as possible to minimize the risk to get to the waypoint as shown in Figure 6.

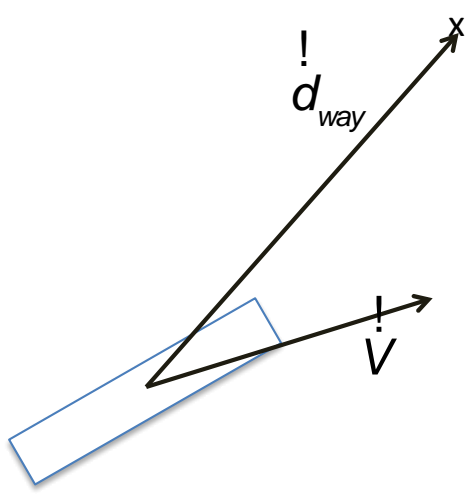

Figure 6. Waypoint geometry. Source: authors.

There are two components to the risk as can be seen. The first is how far away from the waypoint the agent is. This is calculated through equation (3).

$$
P_{T}=1-e^{-\frac{\left|\vec{d}_{\text {way }}\right|}{S_{f}}}
$$

The second part of this is the component of where the agent is likely to go based upon its state geometry. That is calculate that through equation (4).

$$
P_{V}=\frac{1}{2}\left(1-\frac{\vec{V} \cdot \vec{d}_{\text {way }}}{|\vec{V}|\left|\vec{d}_{\text {way }}\right|}\right)
$$

This is then combined through a logical "AND" arrangement to get the probability that the agent will not get to its waypoint (equation (5)).

$$
P_{w}=P_{V}\left(P_{T}+P_{V} \quad P_{V} P_{T}\right)
$$

B. Power Risk 
For nautical systems power generation is a key consideration. By changing the speed of the vessel the decision maker changes the power generation of the ship. The curve that expresses this is shown in Figure 7.

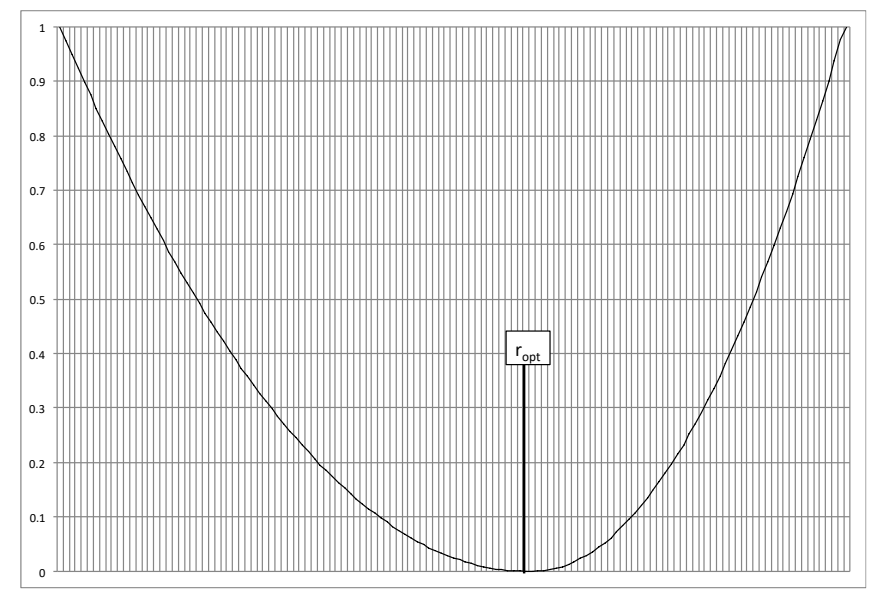

Figure 7. Power setting risk plot. Source: authors.

This is a simple piecemeal equation (6) where the risk of failure occurs at the redline or if the power off is shut off entirely to the system. The minimum risk occurs when the system has the optimal power usage set. The value of the optimal position is vessel dependent. This function is also developed this way such that the derivative is smooth across the break so that gradient based methods have a derivative that exists for all power settings.

$$
\begin{aligned}
& P_{P}=\left\{\frac{r^{2} 2 r_{o p t} r+r_{o p t}^{2}}{r_{\text {max }}^{2} 2 r_{o p t} r_{\text {max }}+r_{o p t}^{2}} \quad r>r_{o p t}\right. \\
& \square \frac{1}{r_{\text {opt }}^{2}} r^{2} \quad \frac{2}{r_{\text {opt }}} r+1 \quad r \square r_{\text {opt }}
\end{aligned}
$$

\section{Roll Risk}

At some point (also vessel specific) the vessel will tip. Ideally the vessel does not have to change direction. This risk is shown graphically in Figure 8.

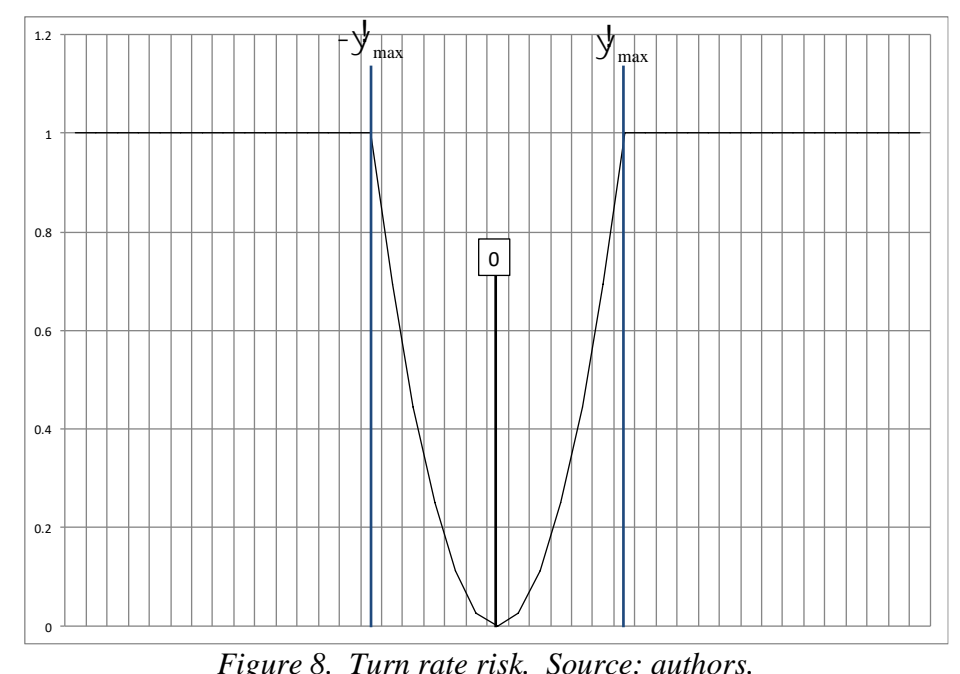

The model for this risk is also piecemeal (equation (7)). Like the equation in Section 3-B, the equation also needs to have a continuous first derivative with a well defined minimum value ( 0 turn rate). 


$$
P_{R}=\left\{\begin{array}{cc}
1 & \dot{\psi}<-\dot{\psi}_{\max } \\
-\frac{1}{\dot{\psi}_{\max }^{2}} \dot{\psi}^{2}-\frac{2}{\dot{\psi}_{\max }} \dot{\psi} & -\dot{\psi}_{\max } \leq \dot{\psi}<0 \\
\frac{2}{\dot{\psi}_{\max }} \dot{\psi}-\frac{1}{\dot{\psi}_{\max }^{2}} \dot{\psi}^{2} & 0 \leq \dot{\psi}<\dot{\psi}_{\max } \\
1 & -\dot{\psi}_{\max } \leq \dot{\psi}
\end{array}\right.
$$

D. Collision Risk

The first step to assess the risk of collision is to linearize the equations of motion so that the agent's system is an approximation of the non-linear dynamics. The coefficients of these matrices $\left({ }_{1}\right.$ and $\left.{ }_{2}\right)$ are determined from the pre-processing of the observed historical data. This allows for a state transition matrix of the form shown in equation (8).

$$
\vec{x}_{k+1}=\phi_{1} \vec{x}_{k}+\phi_{2} \vec{u}_{k}+\vec{\chi}
$$

This gives the system the ability to update the variance of the system through this linearized estimate equation (9). Note that for this simulation the agent has the knowledge of the command variance. However, there are other implementations [Williams and Jin, 2019] that don't assume or have data directly on this. In these cases other variance updates are used.

$$
k_{k+1}={ }_{1} \quad k 1^{T}+{ }_{2} \quad u^{T}
$$

For computational efficiency a multivariate Gaussian distribution (equation (10)) is used that is the sum of multiple Gaussian distributions (equation (11)). Details of how these variances are determined are outside the scope of this specific paper [Williams and Jin, 2019], but are discussed elsewhere.

$$
\begin{gathered}
P_{C}^{i}=\int_{-\infty}^{\infty} \int_{-\infty}^{\infty} \cdots \int_{-a}^{a} \int_{-b}^{b} \frac{1}{(2 \pi)^{d}\left|\Sigma_{i}\right|^{\frac{d}{2}}} e^{\left(-\frac{1}{2}(\vec{x}-\vec{\mu})^{T} \Sigma^{-1}(\vec{x}-\vec{\mu})\right)} \\
P_{C}=\frac{a_{i=1} P_{C}^{i}}{a_{i}}
\end{gathered}
$$

For multiple TargetShips collisions cannot be added since they are not independent events. For the same reason the probability of any collision is not the multiplication of these non-independent events. However, misses are each independent events. Thus, to determine the probability of all collisions the system takes the probability of all misses and then perform a logical "NOT" (equation (12)).

$$
\left.P_{\text {Col }}=1{\underset{i=1}{n_{\text {targ }}}(1}_{(1} P_{C_{O>i}}\right)
$$

In the determination of each individual probability (10) there are integrals with infinite bounds. These are all the non-position (physical). The infinite bounds are there to denote that the system does not need to know any specific non-location state. The only concern that the agent has is when the positions overlap. The velocity and orientation (for instance) can be any value. 


\section{E. Trajectory Risk}

To calculate the risk along the trajectory the sytem starts with a discretized command set that allows it to calculate OwnShip trajectory. It then estimates the average trajectory (for each Gaussian component), $\vec{\mu}$, for each TargetShip.

Along this trajectory at each time step the SRA determines the instantaneous risk (equation (13)). This is the value of the risk at that specific instance of time calculated from the component risk pieces (which are what was defined as the OwnShip objectives).

$$
R(\vec{x}, t)=1-\left(1-P_{C O L}(\vec{x}, t)\right)\left(1-P_{R}(\vec{x}, t)\right)\left(1-P_{P}(\vec{x}, t)\right)\left(1-P_{W}(\vec{x}, t)\right)
$$

This instantaneous risk is integrated across the projected trajectory (equation (14)). A discount factor is included in this integral since the further in time we go, the less valuable those values are.

$$
R(\vec{u})=\frac{r}{1-e^{-r t_{f}}} \int_{\tau=0}^{t_{f}} e^{-r \tau} R_{i n s t}(\tau, \vec{x}(\vec{u}, \tau)) d \tau
$$

\section{SIMULATION RESULTS}

A set of standard scenarios are used to test ship captains on their ability to avoid collisions when confronted with dangerous situations. These set of 17 scenarios have been used by the iSC team to test the efficiency of updates to the nautical collision avoidance algorithms. This set is shown in Table 1, and locations are provided in the NED frame. The scenarios that are highlighted in yellow are those that will be presented. All of these scenarios were run and verified, these are chosen solely for aesthetic purposes.

\begin{tabular}{|c|c|c|c|c|c|c|c|c|c|}
\hline $\begin{array}{c}\text { Scenario } \\
\#\end{array}$ & $\begin{array}{c}\text { Bearing } \\
\text { (deg) }\end{array}$ & $\begin{array}{c}\text { Distance } \\
\text { (nmi) }\end{array}$ & $\mid \begin{array}{c}\text { Course } \\
\text { (deg) }\end{array}$ & $\begin{array}{c}\text { Speed } \\
\text { (kts) }\end{array}$ & $\begin{array}{c}\text { Scenario } \\
\#\end{array}$ & $\begin{array}{c}\text { Bearing } \\
\text { (deg) }\end{array}$ & $\left|\begin{array}{c}\text { Distance } \\
(\mathrm{nmi})\end{array}\right|$ & $\mid \begin{array}{c}\text { Course } \\
\text { (deg) }\end{array}$ & \begin{tabular}{|c|} 
Speed \\
(kts)
\end{tabular} \\
\hline 1 & 45 & 2.0 & 270 & 15 & \multirow{3}{*}{13} & 0 & 1.0 & 0 & 8 \\
\hline 2 & 70 & 1.0 & 340 & 15 & & 80 & 0.3 & 0 & 15 \\
\hline \multirow{2}{*}{3} & 315 & 2.0 & 90 & 15 & & 45 & 1.4 & 270 & 15 \\
\hline & 45 & 2.0 & 270 & 15 & \multirow{4}{*}{14} & 315 & 2.0 & 90 & 15 \\
\hline 4 & 0 & 2.0 & 180 & 15 & & 45 & 2.0 & 270 & 15 \\
\hline 5 & 6 & 2.0 & 180 & 15 & & 45 & 3.0 & 270 & 15 \\
\hline 6 & 0 & 1.0 & 0 & 10 & & 45 & 4.0 & 270 & 15 \\
\hline \multirow{2}{*}{7} & 45 & 1.4 & 270 & 15 & \multirow{6}{*}{15} & 45 & 3.0 & 270 & 15 \\
\hline & 0 & 2.0 & 180 & 15 & & 328 & 4.3 & 135 & 15 \\
\hline \multirow{3}{*}{8} & 45 & 1.4 & 270 & 15 & & 333 & $\begin{array}{l}4.5 \\
\end{array}$ & 135 & 15 \\
\hline & 0 & 2.0 & 180 & 15 & & 338 & 4.7 & 135 & 15 \\
\hline & 315 & 1.4 & 90 & 15 & & 343 & 4.9 & 135 & 15 \\
\hline \multirow{2}{*}{9} & 80 & 0.4 & 350 & 15 & & 348 & 5.1 & 135 & 15 \\
\hline & 315 & 3.0 & 90 & 15 & \multirow{5}{*}{16} & 45 & 4.3 & 270 & 15 \\
\hline \multirow{3}{*}{10} & 80 & 0.4 & 350 & 15 & & 37 & 4.5 & 270 & 15 \\
\hline & 315 & 3.0 & 90 & 15 & & 51 & 3.4 & 270 & 15 \\
\hline & 45 & 3.0 & 270 & 15 & & 330 & 6.0 & 135 & 15 \\
\hline \multirow{3}{*}{11} & 80 & 0.3 & 0 & 15 & & 0 & 6.7 & 180 & 15 \\
\hline & 315 & 2.0 & 90 & 15 & \multirow{4}{*}{17} & 80 & 0.5 & 0 & 15 \\
\hline & 0 & 2.0 & 180 & 15 & & 45 & 4.2 & 270 & 15 \\
\hline \multirow[b]{2}{*}{12} & 80 & 0.3 & 350 & 15 & & 0 & 6.0 & 180 & 15 \\
\hline & 280 & 0.3 & 10 & 15 & & 355 & 6.0 & 180 & 15 \\
\hline
\end{tabular}

Table 1. Standard test scenarios. Source: authors

In each of these scenarios if no action is taken a collision will occur. The total risk determined by the system is plotted as a function of time against each scenario. A value of $100 \%$ risk means that one of the goals/objectives has failed. For this problem $100 \%$ risk means that a collision will have occurred. 
The first scenario is a simple short distance crossing. The trajectory for a whole 10 minute trajectory is shown in Figure 9. The associated risk is presented in Figure 10. This is a somewhat simple encounter and the risk avoidance algorithm makes the correct decision and is similar to what a trained ship captain would make.

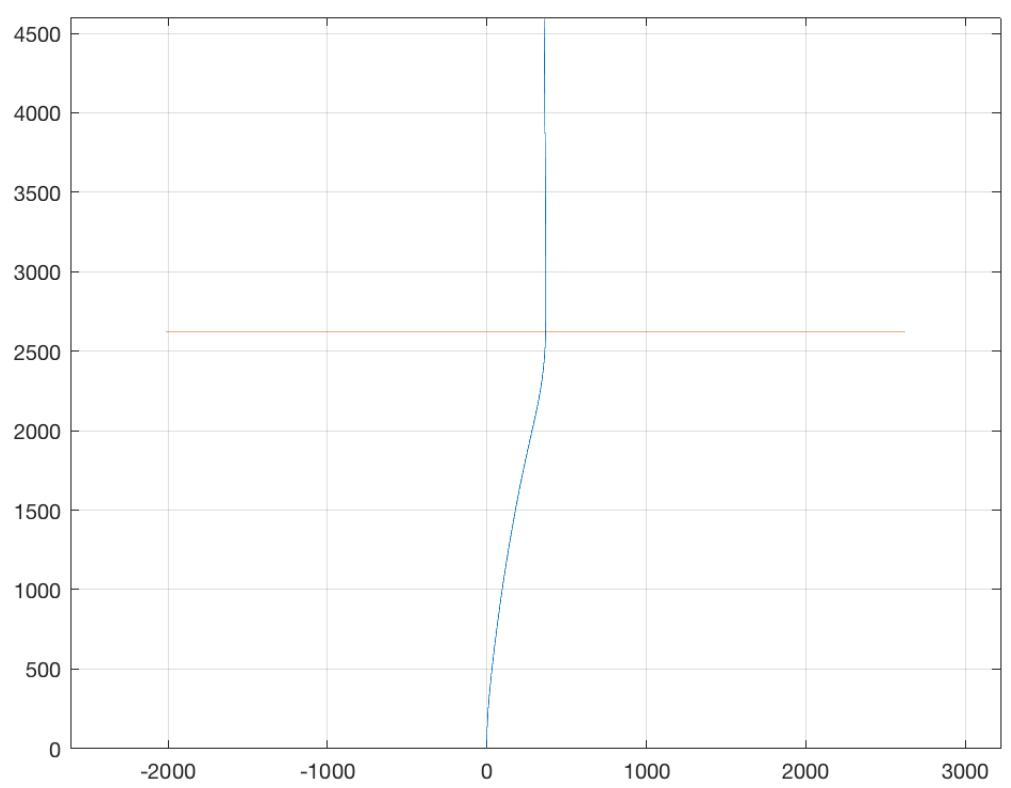

Figure 9. Scenario 1 trajectories. Source: authors.

Scenario 12 is significantly more complicated than the first scenario, and the density of objects in the theater causes the risk to intrinsically increase dramatically. However, as can be seen in Figures 11 and 12 the iSC system does avoid the vessels and follows a path similar to what an experienced ship captain would suggest.

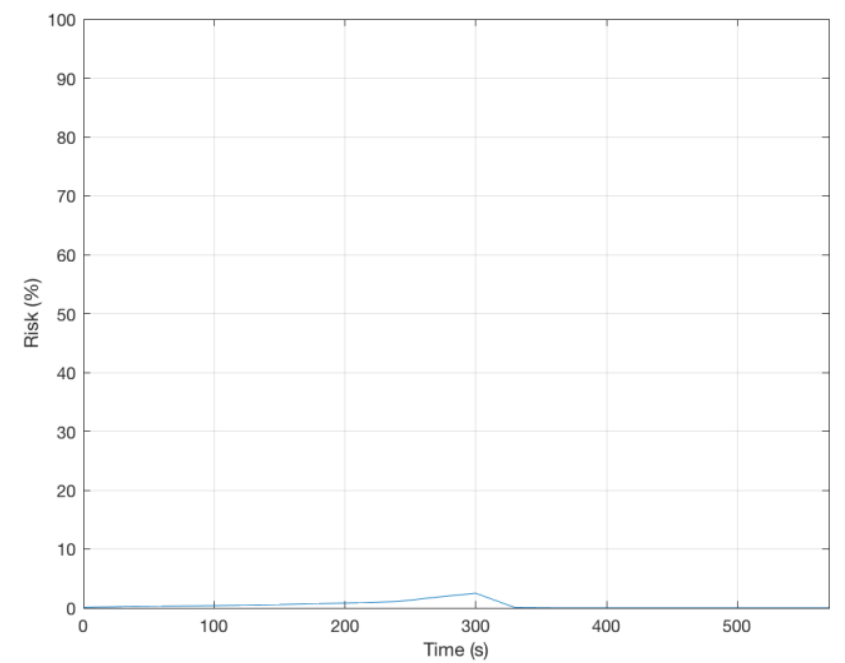

Figure 10. Scenario 1 risk percentage. Source: authors.

Scenario 12 is significantly more complicated than the first scenario, and the density of objects in the theater causes the risk to intrinsically increase dramatically. However, as can be seen in Figures 11 and 12 the iSC system does avoid the vessels and follows a path similar to what an experienced ship captain would suggest. 


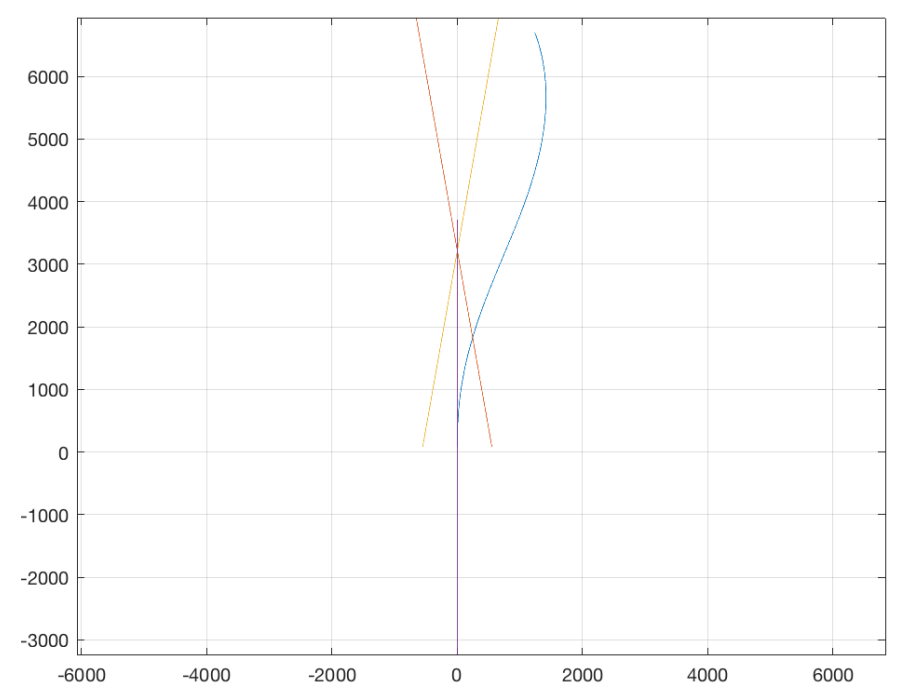

Fig. 11. Scenario 12 trajectories

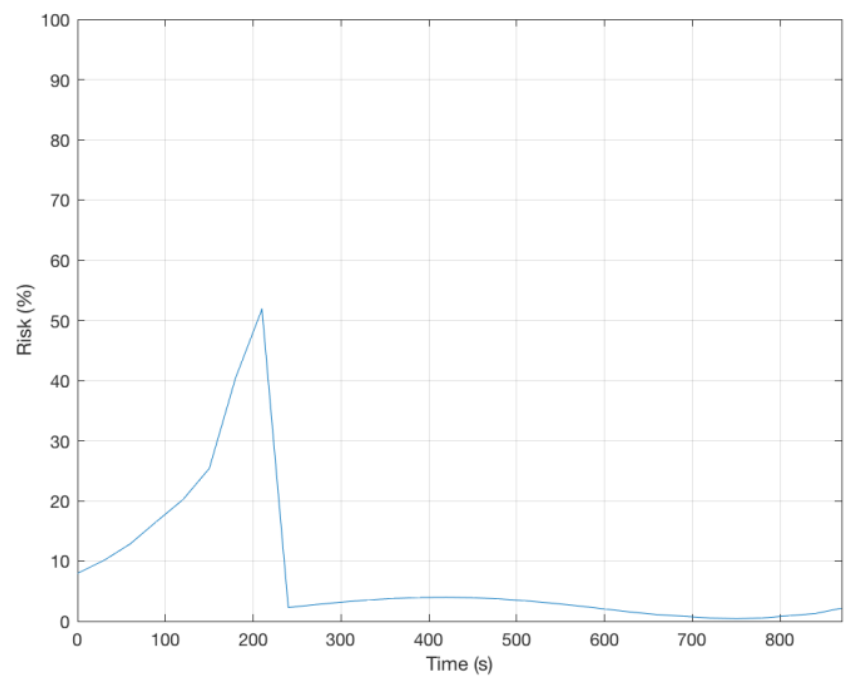

Fig. 12. Scenario 12 risk percentage

The final scenario data presented is 13. This is shown in Figures 13 and 14. Again this shows the system correctly identifying the risks and not following strict COLREGS since that would actually lead to a collision. 


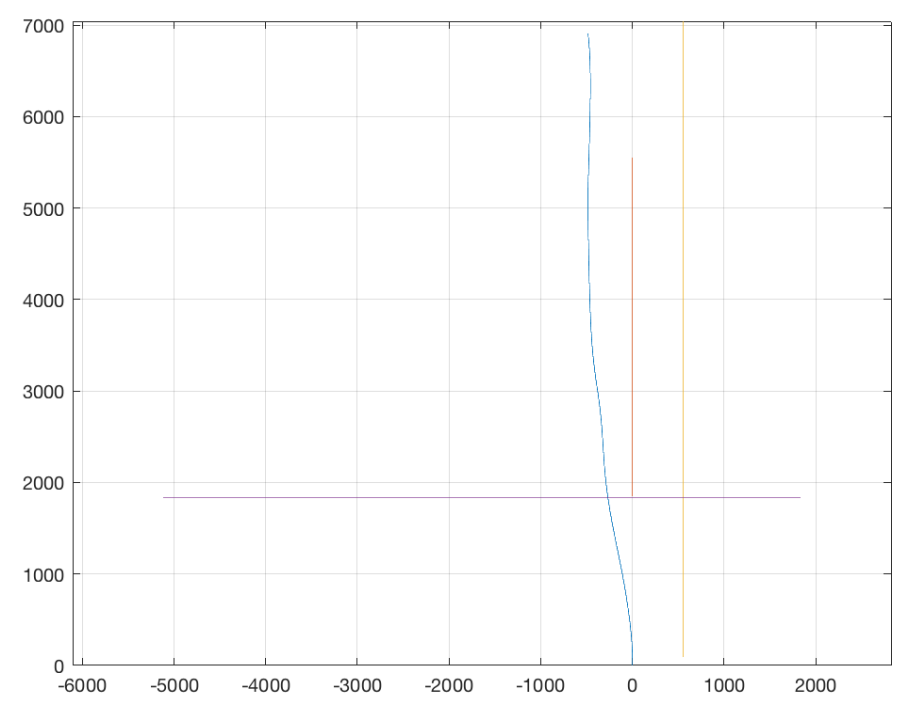

Figure 13. Scenario 13 trajectories. Source: authors.

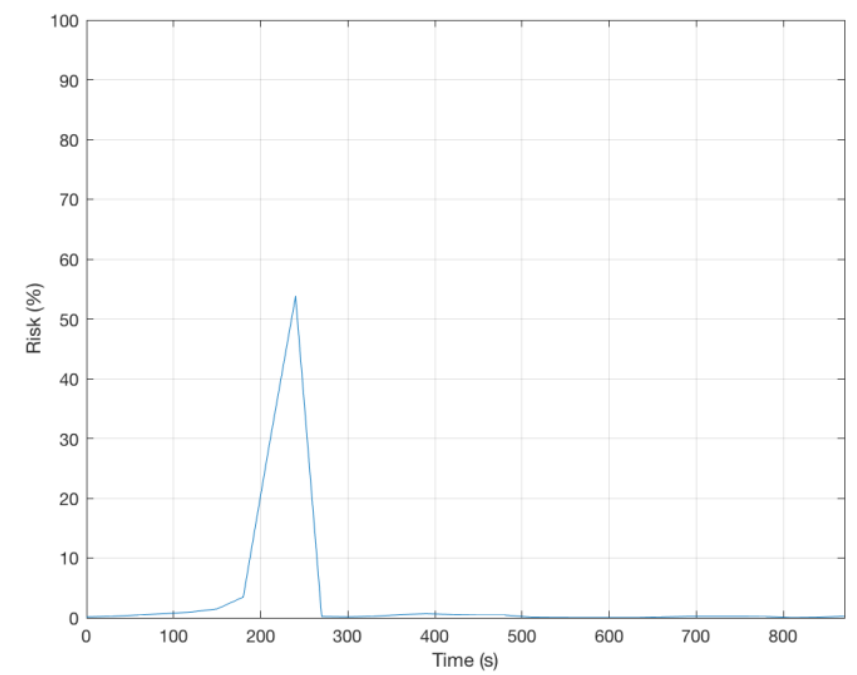

Figure 14. Scenario 13 risk percentage. Source: authors.

It should be noted that in each of these simulation runs, no information is provided that would not be available under normal circumstances. Similarly there is no assumption that these vessels are in any direct communication with each other.

\section{CONCLUSIONS}

The work that has been done on the iSC system has shown that by removing the certainty of the case-bycase decision making intrinsic to traditional guidance solutions typical human captain decisions arise naturally. This does not come from knowing everything that will happen, but instead using data to create a time varying probability field. This field is then used directly to minimize the negative possibilities. Work in this paper and previous work has shown that this is an effective way to provide flexible and safe guidance solutions.

This work is still ongoing and there are still many aspects that need to be developed, most significantly what are the effects of poor or incorrect information. Similarly the work done thus far assumes a fairly simple 
dynamic model is accurate. Work needs to be done to incorporate the probability that the model itself is flawed and thus future state estimates will also be flawed beyond just initial state estimate errors.

\section{ACKNOWLEDGEMENTS}

This research was funded by a grant from Monohakobi Technology Institute; this work would not be possible without their support. The authors also thank Xiongqing Liu, and Karina Hemmendinger for their help in proofreading this document. Additional computational resources were provided by Intel Corporation.

\section{REFERENCES}

[1] Barwise J., "Scenes and Other Situations," The Journal of Philosophy, vol 78, pp. 369-397, Oct. 2006.

[2] Devlin K., "Situation Theory and Situation Semantics", Handbook of the History of Logic, vol 7, pp. 601-664, 2006.

[3] Endsley M., "Toward a theory of situation awareness in dynamic systems," Human Factors: The Journal of the Human Factors and Ergonomics Society, vol 37, pp. 32-64, March 1995.

[4] Endsley M., "Design and Evaluation For situation Awareness Enhancement," Proceedings of the Human Factors Society, pp. 97-101, 1998.

[5] Endsley M., "From Here to Autonomy: Lessons Learned From Human-Automation Research,” Human Factors: The Journal of Human Factors and Ergonomics Socieity, vol 59, pp. 5-27, Feb 2017.

[6] Fossen T., Marine Control Systems: Guidance, Navigation and Control of Ships, Rigs and Underwater Vehicles, Trondheim, Norway: Marine Cybernetics 2002.

[7] Kaiser M., Gans N., and Dixon W., "Vision-based estimation for guidance, navigation, and control of an aerial vehicle," IEEE Transactions on Aerospace and Electronic Systems, vol 46, pp. 1064-1077, August 2010.

[8] Kokar M., Matheus C., and Baclawski K., "Ontology-Based Situation Awareness”, Information Fusion, vol 10, pp. 83-98, Jan 2009.

[9] Kutsuna K., Kuwahara S., Ando H., Nakamura S., and Nakashima T., "Feasibility Study of Automated Process of Ship Navigation A Framework of Action Planning System (APS)", 2018 ICMASS, Korea, 2018.

[10] Lavertsky E., and Wise K. A., Robust and Adaptive Control: with Aerospace Applications, London: Springer-Verlag, 2013.

[11] McHenry R., Long A., Cockrell B., Thibodeau J., and Brand T., "Space Shuttle ascent guidance, navigation, and control," J. of the Astronautical Sciences, vol. 27, pp. 1-38, January 1979.

[12] Ross I. M., and Karpenko M., "Review of Pseudospectral Optimal Control: From Theory to Flight", Annual Reviews in Control, vol 36, pp. 182-197, 2012.

[13] Steinfeldt B., Grant M., Matz D., Braun R., and Barton G., "Guidance, Navigation, and Control System Performance Trades for Mars Pinpoint Landing,” J. of Spacecraft and Rockets, vol 47, pp. 188-198, January 2010.

[14] Ullman D., The Mechanical Design Process, New York, NY: McGraw Hill, 2010.

[15] Vanderplaats G., Numerical Optimization Techniques for Engineering Design, New York, NY: McGraw Hill, 1984.

[16] Williams E., and Jin Y., "Use of Situation and Risk Modeling in Guidance Solutions", IEEE/ION Position Location and Navigation Symposium, 23-26 Apr. 2018. 
[17] Williams E., and Jin Y., "Low Earth Orbit Debris Avoidance Using Situation-Risk Assessment Modeling”, 2018 AIAA SPACE Forum, 2018.

[18] Williams E., and Jin Y., "Development of Probability Fields for Collision Avoidance," currently unpublished. 